\title{
Freeze-Drying as a Novel Biofabrication Method for Achieving a Controlled Microarchitecture within Large, Complex Natural Biomaterial Scaffolds
}

\author{
Claire Brougham \\ Technological University Dublin, claire.brougham@tudublin.ie \\ Tanya J. Levingstone \\ Royal College of Surgeons in Ireland \\ Nian Shen \\ Royal College of Surgeons in Ireland
}

See next page for additional authors

Follow this and additional works at: https://arrow.tudublin.ie/biodevart

Part of the Biomedical Engineering and Bioengineering Commons

\section{Recommended Citation \\ Brougham, C.M., Levingstone, T.J., Shen, N., Cooney, G.M., Flanagan, T.C., Jockenhoevel, S., O'Brien, F.J. (2017). Freeze-Drying as a Novel Biofabrication Method for Achieving a Controlled Microarchitecture within Large, Complex Natural Biomaterial Scaffolds. Advanced Healthcare Materials, vol. 6, no. 21. doi:10.1002/adhm.201700598}

This Article is brought to you for free and open access by the Biomedical Devices and Assistive Technology Research Group at ARROW@TU Dublin. It has been accepted for inclusion in Articles by an authorized administrator of ARROW@TU Dublin. For more information, please contact arrow.admin@tudublin.ie, aisling.coyne@tudublin.ie,gerard.connolly@tudublin.ie.

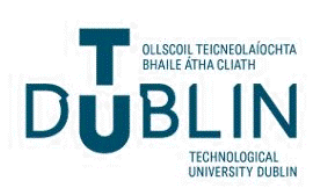




\section{Authors}

Claire Brougham, Tanya J. Levingstone, Nian Shen, Gerard M. Cooney, Thomas C. Flanagan, Stefan Jockenhoevel, and Fergal J. O’Brien

This article is available at ARROW@TU Dublin: https://arrow.tudublin.ie/biodevart/15 


\section{Freeze-drying as a Novel Biofabrication Method for Achieving a}

Controlled Microarchitecture within Large, Complex Natural

\section{Biomaterial Scaffolds}

Claire M. Brougham, Tanya J. Levingstone, Nian Shen, Gerard Cooney, Stefan Jockenhoevel, Thomas C. Flanagan, Fergal J. O’Brien*

Claire M. Brougham, BEng, MEngSc, $\mathrm{PhD}^{1,2,3}$

Tanya J. Levingstone, BEng, MSc, $\mathrm{PhD}^{1,3,4,5}$

Nian Shen, BEng, MSc ${ }^{1,4,6,7}$

Gerard M. Cooney, BEng, MSc, $\mathrm{PhD}^{1,4}$

Thomas C. Flanagan, BSc, $\mathrm{PhD}^{8}$

Stefan Jockenhoevel MD ${ }^{9}$

Fergal J. O’Brien BA, BAI, PhD, FAS, CEng, FIEI ${ }^{1,3,4}$

\section{Affiliations}

${ }^{1}$ Tissue Engineering Research Group, Dept. of Anatomy, Royal College of Surgeons in Ireland, 123 St. Stephen's Green, Dublin 2, Ireland.

${ }^{2}$ School of Mechanical and Design Engineering, Dublin Institute of Technology, Bolton St, Dublin 1, Ireland.

${ }^{3}$ Advanced Materials and Bioengineering Research (AMBER) Centre, RCSI \& TCD.

${ }^{4}$ Trinity Centre for Bioengineering, Trinity College Dublin, Dublin 2, Ireland.

${ }^{5}$ School of Mechanical and Manufacturing Engineering, Dublin City University, Dublin 9, Ireland

${ }^{6}$ Department of Women’s Health, Research Institute of Women's Health, University Hospital of the Eberhard Karls University Tübingen, Germany

${ }^{7}$ Department of Cell and Tissue Engineering, Fraunhofer Institute for Interfacial Engineering and Biotechnology IGB, Stuttgart, Germany

${ }^{8}$ School of Medicine, University College Dublin, Dublin 4, Ireland.

${ }^{9}$ Department of Biohybrid \& Medical Textiles (BioTex) at AME-Helmholtz Institute for Biomedical Engineering and ITA-Institut für Textiltechnik, RWTH Aachen University, Germany

\section{Corresponding author:}

Prof. Fergal O’Brien, Department of Anatomy, Royal College of Surgeons in Ireland, 123 St. Stephen's Green, Dublin 2, Ireland.

Telephone Number: +353 (0)1-402-2149 FAX Number: +353(0)1-402-2355 Email: fjobrien@rcsi.ie

Keywords: freeze-drying, collagen, tissue engineering, scaffolds, heart valves 


\section{WILEY-VCH}

Abstract:

The biofabrication of large scaffolds from natural biomaterials into complex 3D shapes with controllable microarchitecture remains a major challenge. Freeze-drying (or lyophilization) is a technique used to create bioactive scaffolds with a porous architecture and is typically only used to generate scaffolds in planar 3D geometries. Here we report the development of a new biofabrication process to form a collagen-based scaffold into a large, complex geometry which has a large height to width ratio, and a controlled porous microarchitecture. This biofabrication process was validated through the successful development of a heart valve shaped scaffold, fabricated from a collagen-glycosaminoglycan co-polymer. Notably, despite the significant challenges in using freeze-drying to create such a structure, the resultant scaffold had a uniform, homogeneous pore architecture throughout. This was achieved through optimization of the freeze-drying mold and freezing parameters. We believe this to be the first demonstration of using freeze-drying to create a large, complex scaffold geometry with a controlled, porous architecture using natural materials. This study validates the potential of using freeze-drying for development of organ-specific scaffold geometries for tissue engineering applications, which up until now might not have been considered feasible. 


\section{WILEY-VCH}

\section{Introduction}

In the field of tissue engineering (TE), biomaterial scaffolds provide a transitional framework which facilitates the development of new tissue, and the influence of scaffold physical and biological characteristics on this neotissue development is well recognized. Significant research has focused on the optimization of individual scaffold characteristics for particular therapeutic applications; however, where regeneration of a tissue with a complex shape is required, fabrication of a suitable scaffold geometry and microarchitecture presents a challenge. While synthetic biomaterial scaffolds can be fabricated using techniques that involve harsh chemicals and large temperature ranges, many of these same methods cannot be used in the fabrication of natural material scaffolds if bioactivity and functionality of the material is to be preserved ${ }^{[1-4]}$. Thus, the biofabrication of large scaffolds from natural biomaterials into complex 3D shapes with controllable microarchitecture remains a major challenge. Freeze-drying (or lyophilization) is a technique used to create bioactive scaffolds with a porous architecture and is typically only used to generate scaffolds in planar 3D geometries. Here we report the development of a new biofabrication process to form a collagen-based scaffold into a large, complex geometry which has a large height to width ratio, and a controlled porous microarchitecture. This biofabrication process was validated through the successful development of a heart valve (HV) shaped scaffold fabricated from a collagenglycosaminoglycan (CG) co-polymer. Notably, despite the significant challenges in using freeze-drying to create such a structure, the resultant scaffold had a uniform, homogeneous pore architecture throughout. We believe this to be the first demonstration of using freeze-drying to create a large, complex scaffold geometry with a controlled, porous architecture using natural materials. This study validates the potential of using the process for development of many organ-specific geometries for tissue engineering applications, in addition to the utilization of the freeze-drying process for applications which up until now might not have been considered feasible. For example, this technique could be used to create natural biomaterial scaffolds for patient specific geometries, without compromising the bioactivity or regeneration potential of the scaffold.

In the field of TE, freeze-drying has typically been used to produce flat 3D sheets of biomaterials where the final geometry of the biomaterial solution is dictated by the shape of the container or mold in which it is fabricated. It is a well characterized technique for creation of collagen-based biomaterial scaffolds with a porous architecture, while preserving collagen's rich bioactive characteristics. In fact, freeze-drying of collagen-based biomaterials not only 


\section{WILEY-VCH}

ensures retention of bioactivity, but also enhances their ability to regenerate tissue ${ }^{[5-10]}$. In our laboratory, we have focused on further enhancing this regenerative capacity by varying the pore architecture within the collagen scaffold to accommodate cells of different types in order to direct regeneration for specific applications [11]. In addition, macromolecules such as glycosaminoglycans have been incorporated to enhance biological activity ${ }^{[12,9,13]}$ and the addition of bioactive molecules such as recombinant growth factors and nucleic acids using non-viral vectors (creating gene-activated scaffolds) have further improved the regenerative potential and functionality of these freeze-dried scaffolds ${ }^{[8]}$. In spite of these benefits, the potential range of therapeutic applications for flat 3D scaffold sheets is limited. Therefore, this study sought to develop a freeze-drying based biofabrication process for the creation of complex, large geometries. The overall goal of the study was to develop a biofabrication process to create a collagen-based scaffold with a homogenous porous microarchitecture in a large, complex geometry.

A semilunar HV shape was chosen as a model indication as it presented two major challengesa large width to height ratio (25 mm diameter, $45 \mathrm{~mm}$ height) and also, complex leaflet components at the midpoint of the geometry. In order to freeze-dry such a complex structure, complete freezing and drying is critical to ensure the scaffold does not collapse once rehydrated. This is not a trivial issue as the large height to width ratio of the HV geometry impedes both heat transfer through the mold and the biomaterial suspension contained within it during freezing as well as reducing the efficiency of ice crystal sublimation during the drying process. A CG co-polymer suspension was used as CG scaffolds have demonstrated increased regenerative capacity over collagen-only scaffolds ${ }^{[9]}$. In the context of creating a complex scaffold geometry, using a CG rather than collagen alone also adds an extra layer of complexity to achieving a uniform pore architecture. Therefore, the specific aims of this study were to: (1) identify the optimal freeze-drying process parameters to reproducibly produce a CG-HV shaped scaffold by investigating the effect of different mold materials (using a custom designed mold) and different freezing temperatures on the resultant scaffold geometry and microarchitecture and (2) to establish the optimal collagen concentration for a HV shaped geometry, in terms of pore size and mechanical properties.

\section{Results}

The effect of mold materials and final freezing temperature 


\section{WILEY-VCH}

A CG-suspension was created as previously described ${ }^{[14]}$ and pipetted into a custom-made HV shaped mold prior to freeze-drying. Molds from two different materials were created, each with different thermal conductivities, in order to assess the optimal mold material to use for the development of a CG scaffold with a large height to width ratio; an aluminum mold (with a thermal conductivity of $49.9 \mathrm{~W} / \mathrm{m}^{2} \mathrm{~K}$ in air and of $4072.5 \mathrm{~W} / \mathrm{m}^{2} \mathrm{~K}$ in water) and a polyoxymethylene (POM) mold (with a thermal conductivity of $22.3 \mathrm{~W} / \mathrm{m}^{2} \mathrm{~K}$ in air and of 44.6 $\mathrm{W} / \mathrm{m}^{2} \mathrm{~K}$ in water). While aluminum more closely matches the thermal conductivity of the freeze-dryer shelf, POM more closely matches that of the CG-suspension and has previously been used to create fibrin based HVs using an injection molding technique ${ }^{[15]}$. The freezing process was initiated from room temperature and using a cooling rate of $1^{\circ} \mathrm{C}$ per minute, a final freezing temperature of either $-10^{\circ} \mathrm{C}$ or $-40^{\circ} \mathrm{C}$ was achieved. Varying the final freezing temperature affects the microarchitecture within the scaffold ${ }^{[16]}$. The temperature profile within the mold during the freezing process was monitored by using thermocouples placed at multiple positions in the suspension to map the temperature during freezing, and the ability of each mold material to create a CG-HV geometry was assessed. Results showed that the aluminum mold allowed the temperature of the CG-suspension to closely match the shelf temperature, regardless of the final freezing temperature, thus allowing tight control over the freezing process (Fig. 1 A \& B). In contrast, the temperature within the POM mold did not follow the freezedryer shelf temperature; the resultant scaffold showed large, random voids at the top of the HV shape, evidence of an uncontrolled cooling process.

\section{Microarchitecture of freeze-dried HV scaffolds}

Having established the capability of the biofabrication process to create a HV geometry, the microarchitecture of the resultant CG-HV scaffolds was investigated by taking samples from the top, leaflet and bottom positions of each of three scaffolds ( $\mathrm{n}=9$ for each position). The microarchitecture of the CG-HV scaffolds was characterized using Scanning Electron Microscopy (SEM) and a histological technique previously developed in house allowed quantitative measurement of scaffold pore size ${ }^{[14]}$. This revealed that the aluminum mold produced CG-HV scaffolds with a homogenous porous microarchitecture, indicative of a controlled freezing process ${ }^{[17]}$ (Fig. 1 C, D, E, F). A scaffold with larger pores resulted from a final freezing temperature of $-10^{\circ} \mathrm{C}$ and there was uniformity of microarchitecture across the $\mathrm{HV}$ geometry, regardless of position within the scaffold; a pore size of $149.5 \pm 22.6 \mu \mathrm{m}$ at the top of the HV shape, $128.9 \pm 39.3 \mu \mathrm{m}$ at the leaflet position and $143 \pm 27.9 \mu \mathrm{m}$ at the bottom of the $\mathrm{HV}$ shape. The histological images (Fig. $1 \mathrm{C}$, E) are more indicative of the pore sizes 


\section{WILEY-VCH}

found in the scaffolds, as the SEM images show the 3D microstructure and thus the pore size cannot be clearly determined from these images (Fig. 1 D, F). In line with the results above, the microarchitecture produced by freezing the CG-suspension within the POM mold was inconsistent, with a large variation in pore size evident (an average pore size of $601.5 \pm 97.2$ $\mu \mathrm{m}$ was produced at the top of the HV geometry; a pore size of $115.9 \pm 56.6 \mu \mathrm{m}$ at the leaflet position, and a pore size of $30 \pm 15.3 \mu \mathrm{m}$ at the bottom of the HV geometry). Following freezedrying, physical and chemical crosslinking methods were used to increase the stiffness of the CG-HV scaffolds ${ }^{[18]}$. This caused the CG-HV scaffolds to shrink by $\sim 25 \%$.

Final freezing temperature of $-40^{\circ} \mathrm{C}$ as shown in aluminum mold, POM mold \& controlled shelf temperature
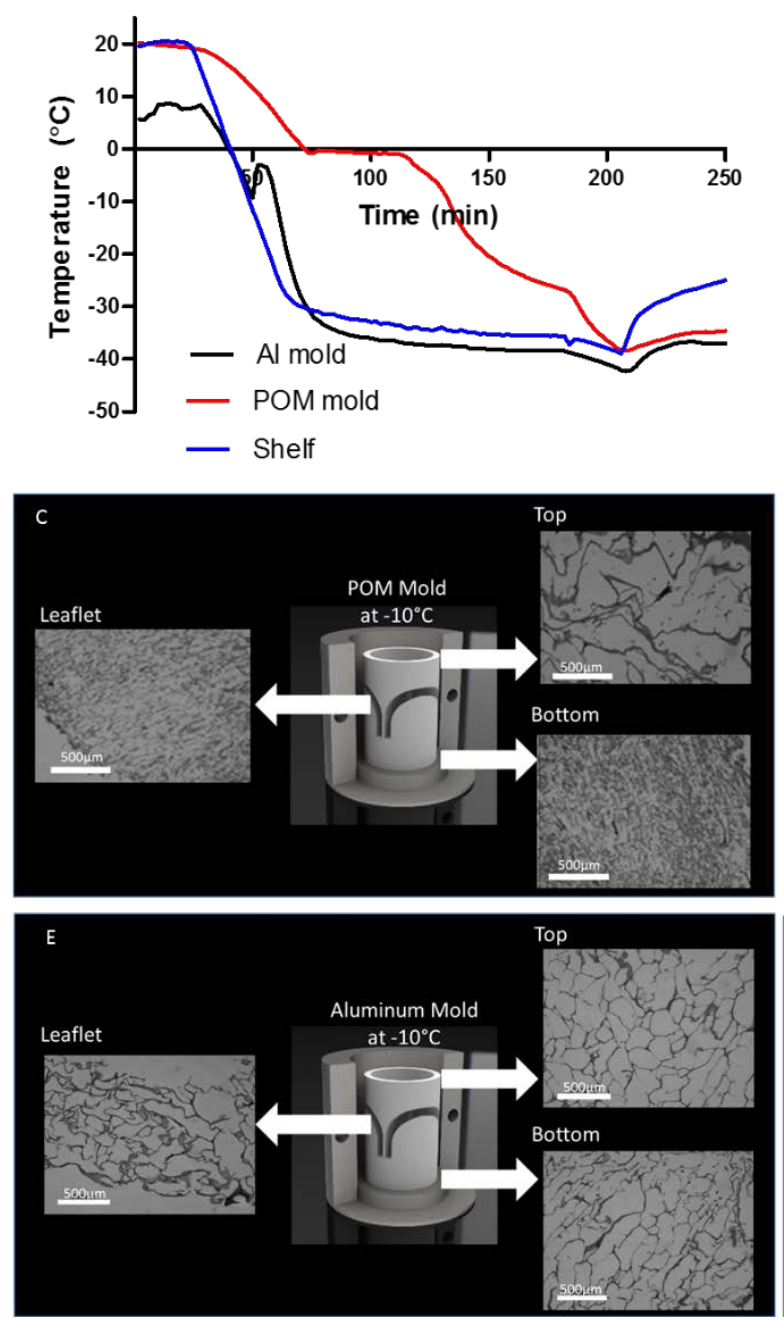

Final freezing temperature of $-10^{\circ} \mathrm{C}$ as shown in aluminium mold, POM mold \& controlled shelf temperature

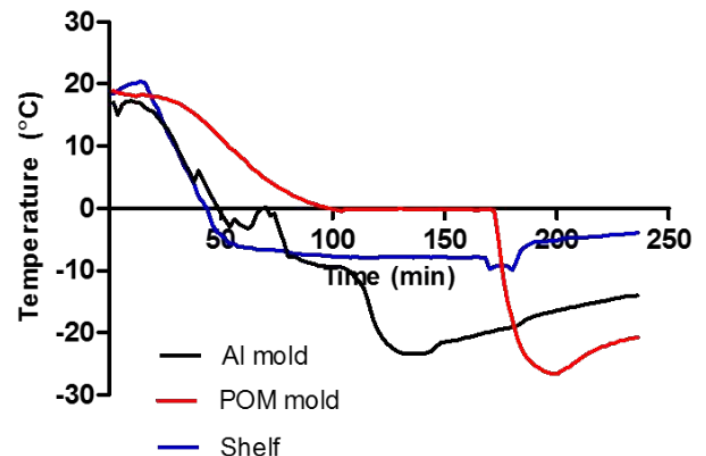

Figure 1 The CG-HV scaffold produced in the aluminum mold was superior to that produced in the POM mold. The average temperature profiles recorded during freezing at a final freezing temperature of $-40^{\circ} \mathrm{C}$ (image A) and $-10^{\circ} \mathrm{C}$ (image B) show that the aluminum mold followed the shelf (reference) temperature more closely than the POM mold. Each line shown is an average of three individual positions (top, leaflet and bottom) within each mold. Image $C$ shows the pore architecture of a CG-HV scaffold fabricated using the POM mold and image $D$ shows the corresponding SEM images. Image E shows the pore architecture of a CG-HV scaffold fabricated using the aluminum mold at a final freezing temperature of $-10^{\circ} \mathrm{C}$ and image $F$ shows the corresponding SEM images. 


\section{WILEY-VCH}

\section{Final freeze-drying mold and freezing parameters}

An optimized final mold was created (fabricated from aluminum with the base and sides of the mold having a thickness of $4 \mathrm{~mm}$ ); this mold differed from the first aluminum prototype as it was upsized to accommodate shrinkage due to the crosslinking techniques used (Fig. 2 A \& B). Based on the results described above, CG-HV scaffolds were fabricated using a cooling rate of $1^{\circ} \mathrm{C} / \mathrm{min}$ and a final freezing temperature of $-10^{\circ} \mathrm{C}$ (Fig. $2 \mathrm{C} \& \mathrm{D}$ ). The dimensions post crosslinking were as required and the leaflets coapted perfectly. The temperature profile within the final mold closely followed the shelf temperature (Fig. 2 E); this control of heat transfer is essential in creating a homogenous pore architecture throughout the CG scaffold. Examination of CG-HV scaffold microarchitecture using both SEM and histology showed an average pore size of $137 \mu \mathrm{m}$ across the HV geometry (an average pore size of $144.8 \pm 23.2 \mu \mathrm{m}$ at the top, the leaflet had an average pore size of $123.2 \pm 14.7 \mu \mathrm{m}$ and the bottom had an average pore size of $145 \pm 10.13 \mu \mathrm{m})($ Fig. 2 F). This final mold, in tandem with the identified process parameters, facilitated repeatable, controlled fabrication of a collagen-based biomaterial into a $\mathrm{HV}$ geometry. The temperature profile observed during the freezing process, the geometry of the scaffold and the resultant homogenous pore architecture, provided validation of this biofabrication process. 


\section{WILEY-VCH}
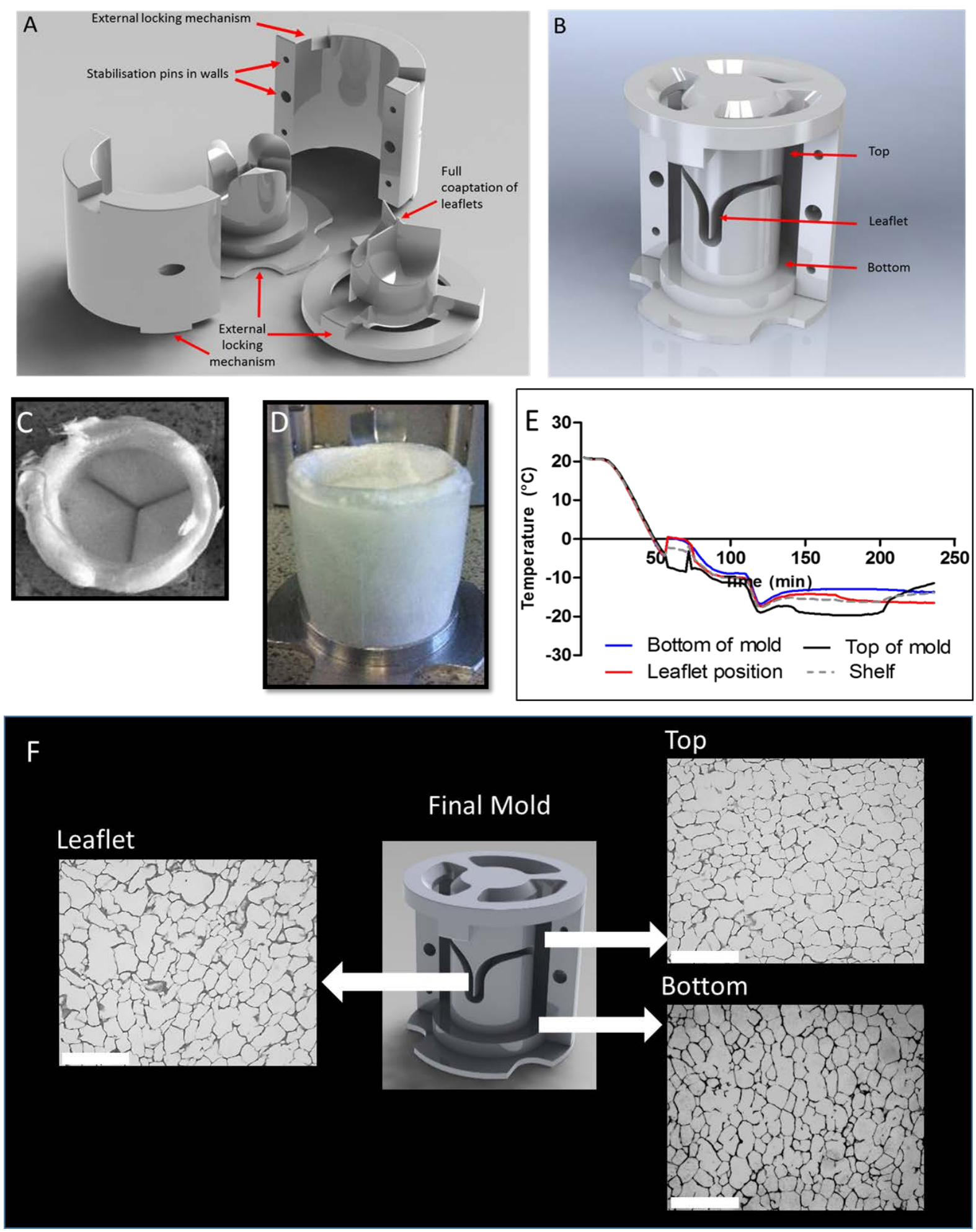

Figure 2 The final mold facilitated fabrication of a CG scaffold in a HV geometry, with macro and microscopic homogeneity. Image A shows an exploded view and image B shows a closed view of the design of the final mold used to produce the CG-HV scaffolds. In image B, the location of the thermocouples for analysis of temperature profile during freeze-drying are indicated. Images C \& D show an example of the CG-HV scaffold produced using the final mold. The graph shown in image E demonstrated that the temperature profile in the final mold showed efficient heat transfer through the mold, with the thermocouples placed at different positions within the mold closely matching the shelf temperature and profile. Image F demonstrated the pore size analysis of the scaffold which was $137 \mu \mathrm{m}$. Scale bar is $500 \mu \mathrm{m}$. 


\section{WILEY-VCH}

Varying collagen concentration for HV applications

Having identified the optimal freeze-drying process parameters to produce a CG-HV scaffold, different concentrations of collagen $(0.5 \%, 0.75 \%$ and $1 \%)$ were used to determine the optimal concentration in terms of scaffold pore size and mechanical properties (the glycosaminoglycan concentration was kept constant at $0.044 \% \mathrm{w} / \mathrm{v}$ ). While no significant differences in pore size were measured in the resultant scaffolds, one of the interesting findings was that the mechanical properties were not linearly related to collagen concentration; the concentration of $0.75 \%$ collagen resulted in a scaffold with the highest compressive (3.83 $\pm 0.45 \mathrm{kPa})$ and tensile modulus (0.59 $\pm 0.04 \mathrm{MPa})$ (Fig. $3 \mathrm{~A} \& \mathrm{~B})$.
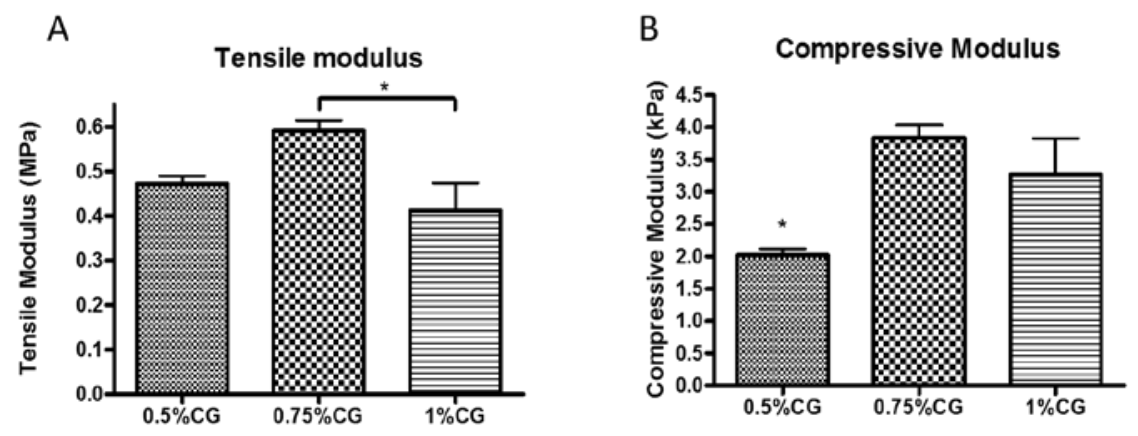

Figure 3 Varying the collagen concentration of CG-HV scaffolds showed that a concentration of $\mathbf{0 . 7 5 \%}$ collagen outperformed $\mathbf{0 . 5 \%}$ and $\mathbf{1} \%$ collagen in terms of tensile and compressive moduli. Image A showed that the only significant increase in tensile modulus was between the $0.75 \%$ collagen and the $1 \%$ collagen concentration. Image B showed there was a significant increase between the compressive modulus of $0.5 \%$ collagen and the moduli of both $0.75 \%$ and $1 \%$ collagen. $(P<0.05)$

\section{Discussion}

Until the development of the biofabrication process described herein, the creation of complex geometries with a controlled porous microarchitecture using natural materials was challenging, especially if the bioactivity of the material was to be retained. Currently, the predominant fabrication methods used for manufacturing complex shapes are 3D printing and electrospinning, and they have significant limitations; 3D printing of natural materials is limited to a resolution of approximately $300 \mu \mathrm{m}^{[19,20]}$ while electrospinning creates smaller scaffolds with an unpredictable pore architecture, where the resultant mechanical properties are anisotropic and there is the added uncertainty as whether the bioactivity of electrospun natural materials is suitably retained. Indeed, this is especially relevant for collagen which is easily denatured into gelatin through electrospinning ${ }^{[1]}$. The freeze-drying process described in this study resulted in the development of a large, complex structure with a homogenous, predefined and inherently repeatable microarchitecture, something which cannot be achieved by 


\section{WILEY-VCH}

electrospinning. Furthermore, pore sizes achieved here are $\sim 137 \mu \mathrm{m}$, and with lower freezing temperatures pore sizes of $\sim 80 \mu \mathrm{m}$ can be achieved. The thickness of the struts between these pores can also be manipulated based on collagen concentration. This demonstrates a level of control far superior than can be achieved by 3D printing of natural materials. The pore sizes achieved within the HV shaped CG matrix are comparable to those found in planar 3D CG sheets ${ }^{[11,21]}$ which have facilitated successful long term culture of cells and regeneration of bone and cartilage in vivo ${ }^{[6,22,23]}$, proving that scaffold pore sizes in this range provide a suitable environment for cell infiltration, attachment and differentiation as well as adequate diffusion of nutrients for TE applications. We suggest that the thicker pore walls in the $1 \%$ collagen scaffold group, resulting from the increased amount of collagen present, permitted less alignment of the pores under compression and tension ${ }^{[24]}$. Additionally, the increase in number of collagen crosslinks in the high concentration collagen group may have led to a decrease in elasticity in the $1 \%$ collagen compared to the $0.75 \%$ collagen group. Varying collagen concentration has previously been shown not to affect the $\sim 99 \%$ porosity of CG scaffolds ${ }^{[25]}$ and this was confirmed in this study through pore size analysis, where the high porosity of the CG-HV scaffold was observed. Additionally, the appropriate scaffold stiffness for HV tissue engineering applications has not been conclusively determined, as different cell types have different requirements ${ }^{[26]}$. Studies have shown that valvular interstitial cells have an activated phenotype (indicative of a disease state) when cultured on stiffer 3D matrices ${ }^{[27,28]}$ and that this activation can be reversed when the compressive stiffness of the matrix is reduced ${ }^{[29]}$. The CGHV scaffold presented here had a compressive stiffness of $~ 3.8 \mathrm{kPa}$ which is below the activation range reported for valvular interstitial cells, potentially enabling the culture of a healthy population of these cells and the use of this CG-HV scaffold for HV applications. Taken together, this study has demonstrated a biofabrication process for shaping of natural materials into complex shaped scaffolds with a consistent, controllable microarchitecture, ideally suited for tissue engineering applications.

Freeze-drying is a technique that not only allows the shaping of natural materials into specific geometries with a porous microarchitecture, but also the tailoring of its structural and biological properties. Mechanical properties can be altered by changing the collagen concentration ${ }^{[25]}$, or through incorporation of biomolecules, such as elastin, prior to freeze-drying, to create a biomaterial with improved viscoelastic properties ${ }^{[30]}$. Following freeze-drying, physical and chemical crosslinking techniques can be employed to tailor the stiffness of the scaffold ${ }^{[25,31]}$. As material stiffness can affect critical cell functions such as differentiation and cytoskeleton organization ${ }^{[31,9,29,32-34]}$, easy manipulation of the mechanical properties of a biomaterial is an 


\section{WILEY-VCH}

attractive advantage for tissue engineering applications as it enables modification of the material to suit its selected application. For certain applications, dimensional stability is critical for functionality and hydrogels such as fibrin can be reinforced with a freeze-dried collagen scaffold to create a natural biomaterial composite that is dimensionally stable in contact with contractile cells ${ }^{[18]}$. Freeze-drying has further advantages as a fabrication technique; freezedried collagen based scaffolds have been used as activated platforms for delivery of bioactive molecules such as recombinant proteins and genes to cells ${ }^{[35,36]}$. For TE, the ability for biochemical cues to be locally delivered to cells provides a range of potential uses including encouragement of relevant extra cellular matrix (ECM) deposition, the ability to turn cells from an activated, to a quiescent phenotype over time, the introduction of plasmid DNA into cells in order to enhance protein production or interfering RNA in order to silence specific genes ${ }^{[8,37]}$. Furthermore, freeze-drying potentially allows addition of these factors prior to freezing resulting in a homogenous distribution of $100 \%$ biomolecule incorporation throughout the collagen matrix unlike most fabrication methods which require growth factor addition after fabrication ${ }^{[8,30,38]}$.

We propose that this study has developed a technique that now allows the advantages of freezedrying to be exploited for use in many applications which have not been possible until now. Collagen is the most abundant protein found in the human body, hence the ability to create collagen-based biomaterials of complex shapes and sizes holds great potential for numerous soft and hard tissue applications. One potential application of the biofabrication process outlined here is in the development of scaffolds with organ-specific geometries. Patient MRI or CT scans, could be imported into software such as Mimics ${ }^{\circledR}$, where the patient specific anatomy can be identified and mapped. The freeze-drying mold could then be CNC machined from the 3D model to create patient specific structures. As discovered in the study presented here, this mold should be fabricated from aluminum and have a $4 \mathrm{~mm}$ thick wall and base. (Aluminum is inexpensive and easy to machine into different shapes, this allows for easy production of large, complicated molds.) Having created a patient specific mold this could be used to freeze-dry a collagen-based scaffold, matching the sought after anatomical dimensions. We thus propose that this new biofabrication method for producing porous scaffolds from natural materials potentially allows the fabrication of mechanically appropriate, anatomically correct structures with practically unlimited anatomical features. In addition to therapeutic applications, these scaffolds have significant potential as 3D in vitro models for disease characterization, drug development and testing ${ }^{[39]}$. 


\section{WILEY-VCH}

\section{Conclusion}

In summary, this study presents a biofabrication method for forming of natural materials, demonstrated through production of a large scaffold in a complex HV shape. Through control of parameters such as the mold material and final freezing temperature, a repeatable process for producing complex structures with a homogenous porous microarchitecture has been established. This biofabrication technique expands the possibilities of using natural materials for tissue engineering applications. While this study has provided proof of principle for creation of complex porous collagen biomaterials with a large height to width ratio, this biofabrication method could be used for the fabrication of other natural polymer scaffolds, in an unlimited variety of geometries, providing a novel approach for creation of large complex, porous shapes suitable for a range of applications, which until now were not conceivable.

\section{Experimental Section}

\section{$\underline{\text { Scaffold fabrication }}$}

CG-HV scaffolds were fabricated by freeze-drying in a Virtis Genesis Freeze-dryer (Biopharma, UK) using specifically designed HV molds based on the dimensions of designs by ${ }^{[15,40]}$. CGsuspensions with a concentration of $0.5 \%, 0.75 \%$ or $1 \% \mathrm{w} / \mathrm{v}$ collagen (collagen type 1 bovine; Integra Life Science, Plainsboro, NJ, USA), 0.044\% w/v glycosaminoglycan (chondroitin sulphate; Sigma, Arklow, Ireland) in $0.05 \mathrm{M}$ acetic acid (Fisher Scientific), were produced as described previously ${ }^{[16]}$. Freeze-drying was carried out using a freezing rate of $1^{\circ} \mathrm{C} / \mathrm{min}$, final freezing temperatures of $-10^{\circ} \mathrm{C}$ and $-40^{\circ} \mathrm{C}$, with drying carried out at a vacuum pressure of 200 mTorr over 18 hours.

\section{Thermal conductivity of mold materials on freeze-drying temperature profile}

The effect of the thermal conductivity of mold materials on freeze-drying of large complex structures was examined during the freeze-drying of a 0.5\% CG-suspension. Two custom designed HV molds were fabricated; one composed of a polymer (polyoxymethylene; POM) and the other of aluminum. The temperature profile during freezing was recorded at two minute intervals using thermocouples (type T), and associated Wizard 2.0 software (Virtis) with thermocouple elements placed (1) on the freeze-dryer shelf (2) in the CG-suspension at the top of the molds, and (3) at the position of the leaflet, within each of the molds. Based on results from these experiments, an optimized final mold was created and used in the remaining experiments. 


\section{WILEY-VCH}

\section{Scaffold Crosslinking}

Following freeze-drying, all scaffolds were sterilized and physically crosslinked using a vacuum oven (Vacucell, MMM Group, Munich, Germany) at $105^{\circ} \mathrm{C}, 0.05$ bar for 24 hours ${ }^{\text {[31] }}$. A support structure was designed to provide structural support to the CG-HV scaffold during chemical crosslinking using 1-ethyl-3-(3-dimethylaminopropyl) carbodiimide (EDAC) in combination with $\mathrm{N}$-hydroxysuccinimide (NHS) as described previously ${ }^{[31]}$ with ethanol used as the solvent to increase the number of crosslinks formed ${ }^{[41]}$. EDAC $(6 \mathrm{mM})$ was calculated per mass of collagen, dissolved in ethanol and filter sterilized. A molar ratio of 5:2M EDAC/M N-hydroxysuccinimide (a crosslinking catalyst) was used ${ }^{[31]}$.

\section{$\underline{\text { Assessment of pore architecture }}$}

The pore architecture within the CG-HV scaffolds was examined histologically. Using a biopsy punch, three samples each from the top, leaflet and base positions of the structure were cut, fixed in $10 \%$ formalin solution and dehydrated. Following preparation with a JB-4 glycomethacrylate embedding kit (Polysciences, Inc., Warrington, USA) samples were sectioned using a rotary microtome (Leica RM 2255, Leica, Germany). Slides representing each area of the scaffolds were stained with toluidine blue (1\%; Sigma) and imaged using a microscope (Eclipse 90i, Nikon, Japan) with a digital camera (DS Ri1, Nikon, Japan). The captured images were analyzed using a MATLAB (The Math Works Inc, MA, USA) program specifically developed in our lab to construct a best-fit ellipse for each individual pore and calculate the average pore cross-section from each image ${ }^{[14]}$.

Scanning electron microscopy (SEM) (Hitachi SU6600 VP-SEM; Hitachi High Technologies America Inc., Clarksburg, USA) at an accelerating voltage of $15 \mathrm{kV}$, utilizing the secondary electron detector was used to reveal the microarchitecture at different locations within the CGHV scaffolds. Cross-sections were taken from the top, the leaflets and the base of the valves, formalin-fixed, critically point dried (Quorum E3000 CPD, Quorum Technologies, East Sussex, UK) and sputter coated with gold using a Polaron sputter coater (Quorum Technologies).

\section{$\underline{\text { Mechanical properties of different collagen concentrations }}$}

Uniaxial compressive and tensile tests were performed to examine the effect of different collagen concentrations $(0.5 \%, 0.75 \%$ and $1 \%$ CG) on the mechanical properties of $\mathrm{HV}$ leaflets and wall structures. Samples were kept hydrated during testing on a mechanical testing machine (Zwick/Roell, Ulm, Germany) fitted with a $5 \mathrm{~N}$ load cell. Compressive and tensile tests were 


\section{WILEY-VCH}

conducted at a strain rate of $10 \%$ per minute ${ }^{[31,42]}$. The modulus was defined as the slope of a linear fit to the stress-strain curve over $2-5 \%$ strain. Each sample was tested in triplicate.

\section{$\underline{\text { Statistical analysis }}$}

Results are expressed as mean \pm standard deviation. Statistical significance was assessed using one-way analysis of variance (ANOVA), followed by Tukey post-hoc analysis for the pore size analysis experiments and mechanical testing where three individual groups were analyzed. $\mathrm{P}<$ 0.05 values were considered statistically significant. The sample size was $n=3$, where $n$ represents a unique HV scaffold. Pore size analysis and SEM imaging was carried out across multiple samples, from multiple HV scaffolds, at each position evaluated.

\section{$\underline{\text { Acknowledgements }}$}

This study has received funding from the Irish Heart Foundation (Grant number 1045940), the European Research Council under the European Community’s Seventh Framework Programme (FP7/2007-2013) under European Research Council grant agreement no. 239685, and the School of Mechanical and Design Engineering, DIT. The authors would like to acknowledge Dr. Anne Shanahan of the FOCUS Institute, DIT for SEM work and Allison Cudworth.

\section{References:}

[1] D. I. Zeugolis, S. T. Khew, E. S. Y. Yew, A. K. Ekaputra, Y. W. Tong, L.-Y. L. Yung, D. W. Hutmacher, C. Sheppard, M. Raghunath, Biomaterials 2008, 29, 2293.

[2] S. J. Hollister, Nat. Mater. 2005, 4, 518.

[3] M. J. W. Koens, P. J. Geutjes, K. a Faraj, J. Hilborn, W. F. Daamen, T. H. van Kuppevelt, Tissue Eng. Part C. Methods 2011, 17, 327.

[4] T. J. Hinton, Q. Jallerat, R. N. Palchesko, J. H. Park, M. S. Grodzicki, H.-J. Shue, M. H. Ramadan, A. R. Hudson, A. W. Feinberg, Sci. Adv. 2015, 1, e1500758.

[5] T. J. Levingstone, A. Matsiko, G. R. Dickson, F. J. O’Brien, J. P. Gleeson, Acta Biomater. 2014, 10, 1996.

[6] F. David, T. J. Levingstone, W. Schneeweiss, M. de Swarte, H. Jahns, J. P. Gleeson, F. J. O’Brien, J. Tissue Eng. Regen. Med. 2015, DOI 10.1002/term.2006.

[7] J. D. Stack, T. J. Levingstone, W. Lalor, R. Sanders, C. Kearney, F. J. O’Brien, F. David, J. Tissue Eng. Regen. Med. 2016, DOI 10.1002/term.2173.

[8] R. M. Raftery, D. P. Walsh, I. M. Castaño, A. Heise, G. P. Duffy, S.-A. Cryan, F. J. O’Brien, Adv. Mater. 2016, 28, 5447.

[9] I. V Yannas, D. S. Tzeranis, B. A. C. Harley, P. T. C. So, Philos. Trans. A. Math. Phys. Eng. Sci. 2010, $368,2123$. 


\section{WILEY-VCH}

[10] T. M. Freyman, I. V Yannas, L. J. Gibson, Prog. Mater. Sci. 2001, 46, 273.

[11] C. M. Murphy, F. J. O’Brien, Cell Adh Migr 2010, 4, 377.

[12] R. A. Hortensius, B. A. C. Harley, Biomaterials 2013, 34, 7645.

[13] S. R. Caliari, B. A. C. Harley, Biomaterials 2011, 32, 5330.

[14] M. G. Haugh, C. M. Murphy, F. J. O’Brien, Tissue Eng Part C Methods 2010, 16, 887.

[15] S. Jockenhoevel, K. Chalabi, J. S. Sachweh, H. V Groesdonk, L. Demircan, M. Grossmann, G. Zund, B. J. Messmer, Thorac. Cardiovasc. Surg. 2001, 49, 287.

[16] F. J. O’Brien, B. A. C. Harley, I. V Yannas, L. J. Gibson, Biomaterials 2005, 26, 433.

[17] F. J. O’Brien, B. A. C. Harley, I. V Yannas, L. Gibson, Biomaterials 2004, 25, 1077.

[18] C. M. Brougham, T. J. Levingstone, S. Jockenhoevel, T. C. Flanagan, F. J. O’Brien, Acta Biomater. 2015, 26, 205.

[19] H.-W. Kang, S. J. Lee, I. K. Ko, C. Kengla, J. J. Yoo, A. Atala, Nat. Biotechnol. 2016, 34, 312.

[20] M. K. Włodarczyk-Biegun, A. del Campo, Biomaterials 2017, DOI 10.1016/j.biomaterials.2017.04.019.

[21] B. A. C. Harley, H.-D. Kim, M. H. Zaman, I. V Yannas, D. A. Lauffenburger, L. J. Gibson, Biophys. J. 2008, 95, 4013.

[22] T. J. Levingstone, A. Ramesh, R. T. Brady, P. A. J. Brama, C. Kearney, J. P. Gleeson, F. J. O’Brien, Biomaterials 2016, 87, 69.

[23] T. J. Levingstone, E. Thompson, A. Matsiko, A. Schepens, J. P. Gleeson, F. J. O’Brien, Acta Biomater. 2015, 32, 149.

[24] B. A. C. Harley, J. H. Leung, E. C. C. M. Silva, L. J. Gibson, Acta Biomater. 2007, 3, 463.

[25] C. M. Tierney, M. G. Haugh, J. Liedl, F. Mulcahy, B. Hayes, F. J. O’Brien, J. Mech. Behav. Biomed. Mater. 2009, 2, 202.

[26] S. Jana, B. J. Tefft, D. B. Spoon, R. D. Simari, Acta Biomater. 2014, 10, 2877.

[27] K. M. Mabry, S. Z. Payne, K. S. Anseth, Biomaterials 2016, 74, 31.

[28] N. Latif, A. Quillon, P. Sarathchandra, A. McCormack, A. Lozanoski, M. H. Yacoub, A. H. Chester, PLoS One 2015, 10, e0127844.

[29] A. M. Kloxin, J. A. Benton, K. S. Anseth, Biomaterials 2010, 31, 1.

[30] A. J. Ryan, F. J. O’Brien, Biomaterials 2015, DOI 10.1016/j.biomaterials.2015.09.003.

[31] M. G. Haugh, C. M. Murphy, R. C. McKiernan, C. Altenbuchner, F. J. O’Brien, Tissue Eng. Part A 2011, 17, 1201.

[32] C. M. Murphy, A. Matsiko, M. G. Haugh, J. P. Gleeson, F. J. O’Brien, J. Mech. Behav. Biomed. Mater. 2012, 11, 53.

[33] A. J. Engler, S. Sen, H. L. Sweeney, D. E. Discher, Cell 2006, 126, 677.

[34] S. R. Caliari, D. W. Weisgerber, W. K. Grier, Z. Mahmassani, M. D. Boppart, B. A. C. Harley, Adv. Healthc. Mater. 2015, 4, 831.

[35] I. M. Castaño, C. M. Curtin, G. Shaw, J. Mary Murphy, G. P. Duffy, F. J. O’Brien, J. Control. Release 2015, 200, 42.

[36] R. M. Raftery, E. G. Tierney, C. M. Curtin, S.-A. Cryan, F. J. O’Brien, J. Control. Release 2015, DOI 10.1016/j.jconrel.2015.05.005.

[37] K. A. Fitzgerald, J. Guo, R. M. Raftery, I. M. Castano, C. M. Curtin, M. Gooding, R. Darcy, F. J. O’Brien, C. M. O’ Driscoll, Int. J. Pharm. 2016, 511, 1058.

[38] E. Quinlan, A. López-Noriega, E. M. Thompson, A. Hibbitts, S.-A. Cryan, F. J. O’Brien, J. Tissue Eng. 


\section{WILEY-VCH}

Regen. Med. 2015, DOI 10.1002/term.2013.

[39] A. J. Ryan, C. M. Brougham, C. D. Garciarena, S. W. Kerrigan, F. J. O’Brien, Drug Discov. Today 2016, 21, 1437.

[40] T. C. Flanagan, C. G. Cornelissen, S. Koch, B. Tschoeke, J. S. Sachweh, T. Schmitz-Rode, S. Jockenhoevel, Biomaterials 2007, 28, 3388.

[41] C. P. Barnes, C. W. Pemble, D. D. Brand, D. G. Simpson, G. L. Bowlin, Tissue Eng. 2007, 13, 1593.

[42] B. A. C. Harley, T. M. Freyman, M. Q. Wong, L. J. Gibson, Biophys. J. 2007, 93, 2911. 